Recibido: 06.07.2016. Aceptado: 22.07.2016.

\title{
ARTÍCULO 29
}

"El presente Convenio estará abierto a la firma de los Estados Miembros de la Comunidad Iberoamericana”.

\section{FRANCISCO TRUJILLO PONS}

Personal Investigador Contratado Doctor

Departamento Derecho del Trabajo y Seguridad Social

Universidad Jaume I (Castellón de la Plana, España) 


\section{RESUMEN}

Este precepto recoge el modo en que el Convenio Multilateral Iberoamericano de Seguridad Social, de 10 de noviembre de 2007, estará abierto a la firma de los Estados Miembros de la Comunidad Iberoamericana. Ello, sin embargo, no obsta a que cualquier Estados Iberoamericano que no haya cumplido este requisito, pueda suscribir el mismo con posterioridad.

PALABRAS CLAVES: Seguridad Social, trabajadores migrantes, legislación aplicable, excepciones, acuerdos bilaterales o multilaterales, firma.

\section{ABSTRACT}

This article refers how the Multilateral Ibero-American Agreement on Social Security, of November 10, 2007, will be open for signature by the Member States of the IberoAmerican Community. Anyway, any Ibero-American State that has not complied with this requirement can subscribe it later.

KEYWORDS: Social Security, migrant workers, applicable law, exceptions, bilateral or multilateral agreements, signature. 
SUMARIO

I. EL ARTÍCULO 29 DEL CONVENIO RELATIVO A LA FIRMA

II. LA FIRMA DE ESPAÑA. ANTECEDENTES Y ENTRADA EN VIGOR

III. CONCLUSIONES

e-Revista Internacional de la Protección Social, ISNN 2445-3269. 2017, Vol. II, Nº1

http://dx.doi.org/10.12795/e-RIPS.2017.i01.07

Página 50 


\section{EL ARTÍCULO 29 DEL CONVENIO RELATIVO A LA FIRMA}

En la tarea de analizar exclusivamente la firma del Convenio Multilateral Iberoamericano de Seguridad Social conviene, en primer lugar, enfatizar su artículo 29. Así, siguiendo su literalidad: "el presente Convenio estará sujeto a la firma de los Estados Miembros de la Comunidad" refiriéndose a Estados, obviamente, de la Comunidad Iberoamericana ${ }^{1}$.

En estos términos, no existe impedimento alguno para que los Estados Iberoamericanos que no lo hicieron puedan suscribir el mismo con posterioridad. Dicho en otras palabras, el Convenio aquí analizado es un Convenio abierto, pero siempre limitado a los países de la Comunidad Iberoamericana.

En consonancia con la firma, el Convenio preceptúa conforme a su artículo 30 que el mismo quedará sujeto a ratificación, aceptación o aprobación de los Estados firmantes, quienes depositarán los preceptivos documentos en la Secretaría General Iberoamericana a través de la Organización Internacional de Seguridad Social (cuyas siglas son $\mathrm{OISS}^{2}$ ). Así pues, no es tan solo necesaria la firma por cada Estado Parte sino que, posteriormente, resulta necesario que el Convenio se ratifique en base a los requerimientos exigidos por la legislación de cada país para, de este modo, dotarle de fuerza obligatoria.

En este mismo sentido, hay que traer a colación el artículo 31 titulado "entrada en vigor", más concretamente su apartado primero que es de la siguiente literalidad:

"El Convenio entrará en vigor el primer día del tercer mes siguiente a la fecha en que se haya depositado el séptimo instrumento de ratificación, aceptación, aprobación o adhesión. No obstante, éste producirá efectos entre dichos Estados una vez que el Acuerdo de Aplicación sea suscrito por los mismos".

Como puede observarse, en él se establecen tanto las condiciones para la entrada en vigor del Convenio como para, a partir de esta circunstancia, los efectos que produzcan entre los Estados Parte.

En este sentido, condición de eficacia del Convenio resulta ser que también se haya suscrito por dichos Estados el Acuerdo de Aplicación. Así, precisamente sobre este último Acuerdo, su artículo 33.1 cobra especial importancia, en tanto que en él se señala que entrará en vigor en la misma fecha de la firma, respecto de los Estados en los cuales el Convenio ya se encuentre vigente.

Desde esta misma perspectiva, siguiendo el recorrido del mismo artículo 33 del Acuerdo de Aplicación, para los casos en que al momento de la firma del Acuerdo el Convenio no estuviera vigente, entrarán en vigor simultáneamente ambos documentos en la fecha que entre en vigor el Convenio.

Dicho lo cual, para mostrar el actual estado del Convenio, precisamente merced a los artículos 29 y 30 junto con el 31.2 (entrada en vigor) y el 33.3 del Acuerdo de

\footnotetext{
${ }^{1}$ Aunque no tenga una concreción legal, esta expresión se recoge en la Declaración de Salamanca, aprobada en el marco de la XV Cumbre Iberoamericana de Jefes de Estado y de Gobierno, celebrada en dicha ciudad.

${ }^{2}$ En su página web cuenta con un apartado específico destinado al Convenio Multilateral Iberoamericano de Seguridad Social: http://www.oiss.org/-Convenio-Multilateral-.html.
}

e-Revista Internacional de la Protección Social, ISNN 2445-3269. 2017, Vol. II, Nº1 
Aplicación (entrada en vigor) se puede confrontar el cuadro que se relaciona a continuación $^{3}$ en el que, de un modo sencillo, se señalan a modo de cronograma las siguientes fechas importantes para cada Estado Parte: fecha de la firma del Convenio, fecha de ratificación, fecha de depósito de los instrumentos, fecha de suscripción al Acuerdo de Aplicación y, por último, la fecha de aplicación efectiva del Convenio.

\begin{tabular}{|c|c|c|c|c|c|c|}
\hline $\begin{array}{l}\text { PAISES QUE LO } \\
\text { HAN FIRMADO }\end{array}$ & $\begin{array}{l}\text { FECHA } \\
\text { FIRMAA } \\
\text { CONVENIO }\end{array}$ & $\begin{array}{l}\text { FECHA } \\
\text { RATIFICACION }\end{array}$ & $\begin{array}{c}\text { FECHA } \\
\text { DEPOSITO } \\
\text { INSTRUMENTO } \\
\text { RATIFICACION EN } \\
\text { LA SECIE-OISS }\end{array}$ & $\begin{array}{l}\text { FECHA } \\
\text { SUSCRIPCIÓN } \\
\text { ACUERDO } \\
\text { APLICACION }\end{array}$ & $\begin{array}{l}\text { APLICACIÓN } \\
\text { EFECTIVA } \\
\text { CONVENIO }\end{array}$ & POBLACION(1) \\
\hline Argentina & $10 / 11 / 2007$ & 09/06/2010 & $31 / 05 / 2016$ & $31 / 05 / 2016$ & $1 / 08 / 2016$ & 42.980 .026 \\
\hline [f Bolivia & $10 / 11 / 2007$ & $08 / 11 / 2010$ & $02 / 02 / 2011$ & $18 / 04 / 2011$ & $01 / 05 / 2011$ & 10.027 .254 \\
\hline Brasil & $10 / 11 / 2007$ & $30 / 10 / 2009$ & $11 / 12 / 2009$ & $19 / 05 / 2011$ & $19 / 05 / 2011$ & 201.000 .000 \\
\hline * Chile & $10 / 11 / 2007$ & $18 / 11 / 2009$ & $30 / 11 / 2009$ & $01 / 09 / 2011$ & $01 / 09 / 2011$ & 16.572 .475 \\
\hline I Colombia & $26 / 11 / 2008$ & & & & & \\
\hline Costa Rica & $10 / 11 / 2007$ & & & & & \\
\hline Ecuador & $07 / 04 / 2008$ & $31 / 08 / 2009$ & $04 / 11 / 2009$ & $20 / 06 / 2011$ & $20 / 06 / 2011$ & 14.067 .000 \\
\hline El Salvador & $10 / 11 / 2007$ & $29 / 05 / 2008$ & $04 / 09 / 2008$ & $17 / 11 / 2012$ & $17 / 11 / 2012$ & 6.251 .495 \\
\hline España & $10 / 11 / 2007$ & $05 / 02 / 2010$ & $12 / 02 / 2010$ & $13 / 10 / 2010$ & $01 / 05 / 2011$ & 46.507 .760 \\
\hline Paraguay & $10 / 11 / 2007$ & $15 / 12 / / 2010$ & $09 / 02 / 2011$ & $28 / 10 / 2011$ & $28 / 10 / 2011$ & 6.672 .631 \\
\hline Perú & $10 / 11 / 2007$ & $12 / 09 / 2013$ & $30 / 01 / 2014$ & $20 / 10 / 2016$ & $20 / 10 / 2016$ & 31.915 .789 \\
\hline Portugal & $10 / 11 / 2007$ & $27 / 10 / 2010$ & $22 / 12 / 2010$ & $19 / 03 / 2013$ & $21 / 07 / 2014$ & 10.561 .614 \\
\hline República Dominicana & $07 / 10 / 2011$ & & & & & \\
\hline$\stackrel{\text { a }}{=}$ Uruguay & $10 / 11 / 2007$ & $24 / 05 / 2011$ & $26 / 07 / 2011$ & $26 / 07 / 2011$ & $01 / 10 / 2011$ & 3.286 .314 \\
\hline Venezuela & $10 / 11 / 2007$ & $16 / 02 / 2009^{\star *}$ & & & & \\
\hline
\end{tabular}

(1) Según la Oficina de Información Diplomática 2012.

En efecto, como bien detalla la Oficina de Información Diplomática por medio de este cuadro-resumen, son 15 países los que han firmado el Convenio hasta el momento; muchos de los cuales -entre los que se encuentra España- coincidieron en la fecha de 10/11/2007, siendo Ecuador, Colombia y la República Dominicana quienes, cuatro años después (2011), se unieron a la firma de los restantes países.

\section{LA SITUACIÓN DE ESPAÑA. ANTECEDENTES Y ENTRADA EN VIGOR}

Particularizando en el caso español, nuestro país ratificó el Convenio con fecha de 5 de febrero de 2010 y, conforme indica el mencionado artículo 30, depositó el instrumento de ratificación el 12 de febrero de 2010 ante la Secretaría General Iberoamericana a través de la OISS.

Posteriormente, con fecha de 13 de octubre de 2010, y con el firme propósito de beneficiar a los trabajadores migrantes y sus familias, España procedió a la firma del Acuerdo de Aplicación del Convenio ante el Secretario General de la OISS y el representante del Secretario General Iberoamericano. Fue una ratificación provisional la

${ }^{3}$ Cuadro publicado por la OISS: http://www.oiss.org/Estado-de-Situacion.html. Consultado el 19.03.2017.

e-Revista Internacional de la Protección Social, ISNN 2445-3269. 2017, Vol. II, Nº1

http://dx.doi.org/10.12795/e-RIPS.2017.i01.07

Página 52 
efectuada por España en 2010, por cuanto el Acuerdo firmado en ese año concluye con la siguiente cláusula: "España declara que no puede proceder a la firma definitiva del presente Acuerdo de Aplicación hasta que se hayan cumplido los requisitos establecidos en el ordenamiento interno para la prestación del consentimiento. No obstante, España declara que aplicará el Acuerdo provisionalmente desde el momento de la firma".

El Acuerdo de Aplicación del Convenio Multilateral Iberoamericano de Seguridad Social, que se venía aplicando provisionalmente desde la firma por España con fecha de 13 de octubre de 2010 a través de la Resolución del Ministerio de Asuntos Exteriores de 17 de diciembre de 2010, entró en vigor definitivamente en nuestro país el 27 de diciembre de 2011 (BOE 15 febrero 2012), poniendo de esta manera fin a su aplicación provisional y completando la inserción efectuada en el BOE de 8 de enero de 2011.

\begin{tabular}{|c|c|c|}
\hline Estado Parte & $\begin{array}{c}\text { Manifestación del } \\
\text { Consentimiento }\end{array}$ & Entrada en vigor \\
\hline \multirow{2}{*}{ España } & $13 / 10 / 2010$ firma con & $13 / 10 / 2010$ Aplicación \\
& $\begin{array}{c}\text { reserva de Ratificación. } \\
27-12-2011 \text { Notificación. }\end{array}$ & $\begin{array}{c}\text { provisional. 27-12-2011 } \\
\text { Entrada en vigor. }\end{array}$ \\
\hline
\end{tabular}

Así con todo, la entrada en vigor con carácter general de este Acuerdo se produjo el 1 de mayo de 2011, de acuerdo con lo previsto en su ya mencionado artículo 33, siendo precisamente esa misma fecha en la que entró en vigor en nuestro país el Convenio Multilateral Iberoamericano de Seguridad Social de 10 de noviembre de 2007.

\section{CONCLUSIONES}

Para el caso español, destaca la fecha de 1 de mayo de 2011, por ser entonces cuando entró en vigor el Convenio, el cual sigue las recomendaciones de la Organización Internacional del Trabajo (OIT) de adoptar los medios para que la globalización económica no vaya en detrimento de la protección social.

El Convenio fue ideado como un mecanismo para promover la coordinación normativa en materia de protección social, respetando los Sistemas Nacionales de Seguridad Social de los diferentes Estados Iberoamericanos. Al día de hoy ha sido ha sido ratificado por Argentina, Bolivia, Brasil, Chile, Ecuador, El Salvador, España, Paraguay, Perú y Portugal. El propósito es alcanzar un espacio de protección para más de 600 millones de personas, y así que puedan irse sumando al mismo el resto de países firmantes a medida de que vayan ratificando el Acuerdo.

Es más, a propósito de la importancia que este Convenio supone para España y sus trabajadores afiliados y procedentes de países de Iberoamérica, según datos proporcionados por el Ministerio de Empleo y Seguridad Social a fecha de enero de 2016, del total de trabajadores de extranjeros que cotizan en el Sistema de la Seguridad Social, según procedencia, tras Rumanía (285.290), Marruecos (201.961), China (92.829) e Italia (71.956), se encuentran países como Ecuador (66.265), y en un menor número Bolivia (54.778). Entre estos dos últimos países, más de 100.00 trabajadores podrían verse beneficiados del Acuerdo y del Convenio que se ha analizado en el presente artículo.

e-Revista Internacional de la Protección Social, ISNN 2445-3269. 2017, Vol. II, №1 